\title{
Tecnología y aprendizaje organizacional factores influyentes sobre la satisfacción profesional en residentes de pregrado
}

Technology and organizational learning influential factors on professional satisfaction in undergraduate residents

\author{
Volumen 18, Número 2 \\ Mayo-Agosto \\ pp. 1-23
}

Este número se publica el 1 de mayo de 2018

DOI: https://doi.org/10.15517/aie.v18i2.33167

Jorge Armando López-Lemus

María Teresa De la Garza Carranza

Revista indizada en REDALYC, $\underline{\text { SCIELO }}$

Revista distribuida en las bases de datos:

LATINDEX, DOAJ, REDIB, IRESIE, CLASE, DIALNET, SHERPA/ROMEO, QUALIS-CAPES, MIAR

Revista registrada en los directorios:

ULRICH'S, REDIE, RINACE, OEEI, MAESTROTECA, PREAL, $\underline{\text { CLACSO }}$ 


\title{
Tecnología y aprendizaje organizacional factores influyentes sobre la satisfacción profesional en residentes de pregrado

\author{
Technology and organizational learning influential factors on professional satisfaction in
} undergraduate residents
}

\author{
Jorge Armando López-Lemus ${ }^{1}$ \\ María Teresa De la Garza Carranza²
}

\begin{abstract}
Resumen: En el artículo de investigación, se analizó el uso de la tecnología, el aprendizaje organizacional y su influencia sobre la satisfacción personal de los residentes de pregrado en las empresas industriales. En el diseño y metodología se empleó una muestra 220 residentes de pregrado del estado de Guanajuato, México. El diseño metodológico de la investigación fue cuantitativa, explicativo, observacional y transversal y para ello se emplearon instrumentos que nos permitieron medir las variables seleccionadas. Para probar las hipótesis establecidas, se desarrolló un modelo de ecuación estructural (SEM). A través del modelo SEM, se demuestra que el uso de la tecnología es un factor que influye positiva y significativamente sobre el aprendizaje organizacional de los residentes de pregrado. De igual manera, el uso de la tecnología influye positiva y significativamente sobre la satisfacción personal de los residentes universitarios. Por otro lado, el aprendizaje organizacional influye positiva y significativamente sorbe la satisfacción personal de los pasantes de pregrado en las empresas industriales del estado de Guanajuato, México. Esta investigación promueve una discusión sobre el uso de las tecnologías, el aprendizaje organizacional y la satisfacción profesional de los residentes de pregrado del estado de Guanajuato. El objetivo del presente estudio de investigación fue conocer la influencia que ejerce el uso de la tecnología de los residentes de pregrado y el aprendizaje organizacional sobre su satisfacción personal en la incorporación en el sector industrial.
\end{abstract}

Palabras clave: TIC, aprendizaje, satisfacción profesional, estudiante universitario.

\begin{abstract}
The research article analyzes the use of technology, organizational learning and its influence on the personal satisfaction of undergraduate residents in industrial companies. The design and methodology includes a sample of 220 undergraduate residents from the Guanajuato state, Mexico. The methodological design of the research was quantitative, explanatory, observational and cross-sectional and the instruments used allow the measure of the selected variables. To test the established hypotheses, a structural equation model (SEM) was developed in the research. Through the SEM model, it is demonstrated that the use of technology is a factor that positively and significantly influences the organizational learning of undergraduate residents. Likewise, the use of technology positively and significantly influences the personal satisfaction of university residents. On the other hand, organizational learning positively and significantly influences the personal satisfaction of undergraduate interns in industrial companies in the Guanajuato state, Mexico. This research promotes a discussion on the use of technologies, organizational learning and professional satisfaction of the undergraduate residents of the state of Guanajuato. The objective of the present research study was to know the influence exerted by the technology of the undergraduate residents and the organizational learning on their personal satisfaction in the incorporation in the industrial sector.
\end{abstract}

Keywords: ICT, learning, professional satisfaction, university student.

\footnotetext{
${ }^{1}$ Catedrático de la Universidad de Guanajuato, México. Doctor Administración y

Estudios Organizacionales. Dirección electrónica: Jorge.lemux@hotmail.com

${ }^{2}$ Catedrática del Instituto Tecnológico de Celaya, México. Doctora en Educación.

Dirección electrónica: teresa.garza@itcelaya.edu.mx
}

Artículo recibido: 8 de noviembre, 2017

Enviado a corrección: 10 de abril, 2018

Aprobado: 23 de abril, 2018 


\section{Introducción}

En la actualidad, las organizaciones y el sector empresarial se enfrentan a diversos cambios y necesidades que exige un mercado globalizado. Una de las principales necesidades está enmarcada por el surgimiento y desarrollo tecnológico, del cual, se va introduciendo en las organizaciones a pasos a agigantados, así mismo, es empleada por las propias instituciones empresariales como una ventaja competitiva en el mercado que se desenvuelve la organización.

Asimismo, la vinculación entre las instituciones educativas y el sector empresarial juega un papel importante en el que el efecto es recíproco, por un lado, las empresas se ven beneficiadas en resolver problemas empresariales en el que los alumnos residentes de pregrado emplean la tecnología con la finalidad de ofrecer soluciones a los problemas identificados, generando un aprendizaje a nivel individual, grupal y organizacional. Por otro lado, la mejora en la calidad de los programas educativos que ofrece las universidades a través de la docencia y la investigación influye en el desempeño empresarial (Secretaria de Educación Pública, México (SEP) y Subsecretaría de Educación e Investigación Tecnológica (SEIT), 1997).

Por otro lado, las organizaciones cuentan con una fuerza laboral que, en su mayoría, está fuertemente influenciada por el uso y manejo de la tecnología, en este sentido, diversas instituciones educativas de nivel superior proveen a las empresas una gran cantidad de alumnos residentes, mismos que, desarrollan sus prácticas profesionales y aplican los conocimientos, habilidades en las mismas organizaciones, sin embargo, por la edad que cuentan los residentes son parte de una nueva generación conocida como "millenians". Esta nueva generación de trabajadores presenta retos importantes para los empleadores debido a sus habilidades (Hershatter y Epstein, 2010)

Es evidente que el uso de la tecnología y el aprendizaje organizacional en el sector empresarial resultan ser algunos factores importantes pues por un lado está la influencia que ejerce la globalización a través de la competitividad empresarial y por otro lado la fuerza laboral que está fuertemente influenciada en el uso de la tecnología para ser cada vez más eficiente su labor (Prior, Hubbard y Rai, 2015).

De esta manera, los residentes emplean la tecnología como uno herramienta que les permitirá automatizar, procesos de administración y gestión, así como la promoción de la innovación como también compartir información necesaria para la toma de decisiones, en 
este sentido, la tecnología es una estrategia para que el residente de pregrado logre sus objetivos individuales y grupales (Lee y Kim, 2014), mostrando un rendimiento organizativo. Por lo tanto, el que los residentes logren el éxito a través del alcance de metas establecidas en corto plazo les permitirá generar en ellos una satisfacción profesional.

La presente investigación tiene como objetivo conocer la influencia que ejerce el uso de la tecnología y el aprendizaje organizacional sobre la satisfacción profesional de los residentes de pregrado.

\section{Marco Teórico}

\subsection{Pasantías o Residencias Profesionales}

De acuerdo con la Secretaria de Educación Pública (SEP, 2017) en México, a través de las instituciones educativas de nivel superior, la pasantía o residencia profesional es una estrategia educativa con un enfoque curricular que le permite al estudiante de pregrado desarrollar un proyecto profesional con la finalidad de resolver una problemática de índole social y productiva, cuyo objetivo radica en fortalecer y llevar acabo las competencias profesionales que el alumno adquirió a través de su formación educativa. De acuerdo con Sánchez de Mantrana (2004), esta estrategia de aprendizaje contribuye a desarrollar en los alumnos competencias y habilidades exigidas en el mundo de hoy.

Un residente de pregrado es un alumno universitario que desarrolla un proyecto ante una problemática real de una empresa, es decir, desarrolla una estancia profesional dentro de una organización, aplicando las competencias profesionales que adquirió a través de su formación académica generando experiencia en lo teórico - práctico con la finalidad de concretar, aumentar y ampliar sus competencias. Este enfoque no es nuevo y se ha utilizado en diversas instituciones. Esta estrategia se ha venido modificando a lo que ahora se conoce como "Educación Dual" que ahora se busca implementar en algunas universidades mexicanas (Gamino, Acosta y Pulido, 2018)

La residencia profesional es un proyecto que el alumno desarrolla dentro del sector productivo de manera individual, grupal o interdisciplinaria enfocado hacia las características, necesidades y requerimientos del sector empresarial, organismo 0 dependencia gubernamental. Existen estudios previos en donde se ha explorado los beneficios de la estancia estudiantil (Coco, 2000 y Clark, 2003). De acuerdo con Knouse y Fontenot (2008) este tipo de experiencia ayuda al educando a incrementar su empleabilidad una vez 
egresado y adicionalmente, crea expectativas reales sobre el mundo laboral. Sin embargo, estos autores mencionan que para que exista una experiencia satisfactoria por ambas partes (estudiante y empleador) debe de haber una participación activa de ambas partes donde se deben de fijar expectativas claras.

En este sentido, la residencia profesional resulta ser una estrategia en la que promueve la empleabilidad del estudiante universitario, a través de la identificación y generación de áreas de oportunidad para desarrollar sus competencias profesionales como lo hace otros programas europeos como Word of Work y la Universidad de Zayed (ElTemtamy, O'Neill y Midraj, 2016)

El enfoque principal de los empleadores con respecto a los graduados universitarios radica en exhibir sus habilidades genéricas (Miller, Biggart y Newton, 2013) como el uso de la tecnología a través de la informática y la comunicación, habilidades cognitivas e intelectuales, manejo de personal y capacidad técnica, así como el conocimiento de empresas y organizaciones (El-Temtamy, O'Neill y Midraj, 2016; Rees, Forbes y Kubler, 2006).

Lowden, Hall, Elliot y Lewin, (2011) señaló que los empleadores a través de sus organizaciones esperan que los universitarios de pregrado y graduados cuenten con las competencias tanto técnicas como disciplinarias correspondientes a la carrera profesional que se encuentran estudiando o en su defecto al grado académico adquirido. En este sentido, tanto los estudiantes como los egresados de pregrado deben contar con una gamma de habilidades y atributos amplios como la comunicación, el uso de la tecnología, trabajo en equipo, pensamiento crítico, resolución de problemas y habilidades gerenciales y directivas (El-Temtamy, O'Neill y Midraj, 2016).

Por esta razón las residencias profesionales que desarrollan los universitarios de pregrado son relevantes pues contrae grandes bondades en el desarrollo profesional, pues adquieren experiencia satisfaciendo las necesidades y contribuyendo al crecimiento de las organizaciones, en este sentido, generando una satisfacción de las carreras en Ingeniería en Bioquímica, Electromecánica, Industrial, Informática, Gestión empresarial, Logística y transporte así como de la Licenciatura en Administración de las universidades públicas en el estado de Guanajuato. 


\subsection{Uso de la Tecnología}

En la actualidad, las organizaciones se enfrentan a diversos cambios y transformaciones derivados a la aparición de nuevas necesidades que exige el mercado, es por ello, que la vinculación entre las instituciones educativas y el sector empresarial resulta ser un papel fundamental para que las empresas responda a las nuevas exigencias que el mercado globalizado exige, y por otro lado, resulta ser fundamental para mejorar la calidad de los servicios educativos de las universidades a través de la docencia y la investigación (Quispe, Victorino y Atriano, 2014; SEP-SEIT,1997).

De acuerdo con Quispe, Victorino y Atriano (2014), una residencia profesional es una estrategia educativa de formación curricular, en que le permite al alumno la incorporación en sentido profesional al sector productivo a través de un proyecto profesional con asesoría tanto interna (universidad) como externa (empresa). En estos proyectos profesionales, generalmente se utiliza la tecnología como base para la mejora de algún proceso productivo o de servicios (García, 2013). Asimismo, un residente de pregrado perteneciente a una nueva generación conocida como "millenian", en el que, el uso y manejo de la tecnología para el desarrollo de sus actividades tanto personales como profesionales es indispensable con la finalidad de poder lograr el rendimiento y su satisfacción profesional (Norum, 2003).

Es por ello que nos enfrentamos ante una nueva generación marcada por los avances tecnológicos que influye a una configuración de un mundo globalizado en el que tanto las instituciones educativas como el sector empresarial de todos los sectores van cambiando las formas y maneras en su operatividad para el funcionamiento de las mismas (Assar, 2015), con la finalidad de lograr mantenerse en el mercado en el que se desenvuelve la firma empresarial u organizacional (De la Garza, Zavala y López-Lemus, 2016). En este sentido, las organizaciones se han enfrentado cambios generados a partir del surgimiento una globalización marcada a partir del surgimiento de la tecnología (Hirsch, Rodríguez y Manríquez, 2015).

Uno de los principales factores que influyen en el aprendizaje de las organizaciones radica en gran parte en el desempeño a nivel individual como en lo grupal de las personas que forman parte de la organización (De la Garza, Zavala y López-Lemus, 2017; TrigueroSánchez y Peña-Vinces, 2013). Por lo tanto, para que las organizaciones puedan hacer frente a la competencia que cada vez es más creciente y responder a las exigencias que se van presentando ante una economía nacional e internacional generada a través de una 
globalización, deberán hacer énfasis en el uso, manejo y aplicación de la tecnología de la información y comunicación (TIC), pues la tecnología representa uno de los factores principales para dar respuesta a las necesidades y demandas que exige la globalización (Gómez, 2011; Hirsch et al. 2015; López-Lemus, De La Garza y Zavala, 2016) y representa una ventaja competitiva (Porter, 2001).

Ante estos nuevos escenarios de los cuales se enfrentan las organizaciones y el sector empresarial ante un rápido crecimiento y desarrollo tecnológico, nos enfrentamos a una brecha digital en las que, una parte de la fuerza laboral tiene conocimiento y hace uso de la tecnología con la finalidad de emplearla para ser cada vez más eficaz y eficiente su trabajo y contribuir en un mejor rendimiento organizacional, dándole sustentabilidad a la micro, pequeña y mediana empresa (MiPyMES), considerando que el uso de la tecnología en las organizaciones resulta ser una ventaja competitiva en el mercado en el que se desenvuelven (Compaine, 2001; Gálves et al., 2014; Lissitsa y Kol, 2016; Porter, 2001).

Asimismo, surge otro factor que ejerce una influencia del uso de la tecnología marcada a través de la fuerza laboral que actualmente cuentan las organizaciones y el sector empresarial. En este sentido, las personas que colaboran para las empresas, en su mayoría, son pertenecientes a una generación conocida como Millenians (Gen Y) (Norum, 2003), estas personas, son introducidas a las empresas, específicamente del sector industrial, ya que cuentan con los conocimientos y habilidades del uso y manejo de las TICs en las que son empleadas para automatizar procesos y hacer más eficiente su rendimiento no solamente en lo individual y grupal a través de los equipos de trabajo, sino también organizacional.

Por lo tanto, la fuerza laboral que pertenece a esta generación, conocida como millenians, hace referencia a las personas que nacieron entre los años 1980 a 1999, esta generación está fuertemente influenciada por el uso y manejo de la tecnología (Gurau, 2012; Norum, 2003; Lissitsa y Kol, 2016; Hofstede, 1980), misma que, es empleada no solamente como una herramienta para comunicarse con los demás, sino también, para emplearla como una estrategia que les permite automatizar, e innovar proceso administrativos y de gestión empresarial que contribuya a la mejora en la productividad, eficiencia y eficacia de la organización (Gursoy et al., 2013; Kupperschmidt, 2000).

En base a lo anterior, el uso y manejo de la tecnología en las organizaciones, así como en el sector empresarial, representa una forma de vida para realizar las actividades y 
funciones tanto de administración como de gestión empresarial para la Gen Y o millenians, considerando las próximas generaciones. Esto es relevante en nuestros días, ya que los millenians se van introduciendo de manera gradual a posiciones jerárquicos altos (Gursoy, Geng-Qing y Karadag, 2013; Kupperschmidt, 2000) y son los responsables de generar una nueva cultura influenciada a través del uso y manejo de la tecnología.

Otro de las características de los milenians radica en compartir con las mismas necesidades en el uso y manejo de la tecnología, así como las ideas, valores, creencias y viven experiencias comunes a través de eventos sociales y económicos en un nivel macro (Strauss y Howe, 1991; Parment, 2013), por consecuente, tienden a buscar las mismas necesidades para generar en ellos una satisfacción profesional.

Por lo tanto, la tecnología se va introduciendo fuertemente en las organizaciones como también el sector empresarial, por un lado, se ve obligada al uso y manejo de la tecnología por la globalización de la cual es participe y por otro lado la fuerza laboral en la que las empresas del sector industrial se encuentran influenciadas por la tecnología. Sin embargo, el contar con personal que cuente con las habilidades del uso y manejo de tecnología y el que una empresa del sector industrial introduzca tecnología para mejorar sus procesos de administración y gestión empresarial, así como también, promover la innovación, representa una ventaja competitiva que ejerce las organizaciones ante un escenario llamado globalización.

\subsection{Aprendizaje organizacional}

De acuerdo con Senge (1990), considera que el aprendizaje organizacional es aquella que expande continuamente la capacidad de crear nuevas experiencias, generando nuevas aspiraciones de manera colectiva y en donde las personas que colaboran dentro de las organizaciones continuamente aprenden a aprender juntas (Bontis, Crossan y Hulland, 2002). Para Kim (1993), considera que el aprendizaje organizacional es la capacidad de la organización para tomar acciones y medidas efectivas.

Asimismo, LeBrasseur, Whissell y Ojha, (2002) consideran que el aprendizaje organizacional implica crear nuevos enfoques para la resolución de problemas con la finalidad de mejorar los procesos, reglas y regular comportamientos dentro de la organización (Barakat y Moussa, 2014; Wong, 2001). En este sentido, el aprendizaje organizacional representa una ventaja competitiva (Hong, 1999; O’Keeffe, 2003). 
A partir de estas definiciones, consideramos que el aprendizaje organizacional es un proceso en el que se desarrollan nuevos conocimientos, procedimientos, reglas y comportamientos para identificar y resolver problemas que enfrenta la organización. Este proceso incluye a tres entidades principales en nuestro caso de estudio: el residente profesional, el encargado del residente por parte de la empresa y la organización que recibe al estudiante

Por lo tanto, los estudiantes residentes juegan un papel importante en el proceso del aprendizaje organizacional, ya que ellos son algunos que se encargan de identificar alguna problemática, es decir, saber por qué (know-why) y resolverla a través de saber el cómo (know-how) empleando sus conocimientos adquiridos en su formación universitaria, y con ello, para dar solución y estrategias para hacer crecer a la organización (Kim, 1993). En este sentido, los alumnos materializan el conocimiento de nivel organizacional a nivel individual por medio de la experiencia (Antonacopoulou, 2006; Kim, 1993) soportado a través de la retroalimentación (feed-back) (Bontis, Crossan y Hulland, 2002) adquiridos a través de la residencia profesional.

De acuerdo con Bontis, Crossan y Hulland (2002) y Kim (1993), el aprendizaje organizacional es multinivel conformado desde tres perspectivas: (1) Aprendizaje a nivel individual, (2) a nivel grupal y (3) a nivel organizacional. En este sentido, estos niveles de aprendizaje tienen vinculaciones e interacciones entre los tres niveles, es decir, el aprendizaje interacciona desde el nivel individual a lo grupal, de lo grupal a lo organizacional y viceversa (Antonacopoulou, 2006).

Para el desarrollo de la presente investigación, se consideró el aprendizaje a nivel organizacional, pues la intensión radica en conocer la influencia que ejerce el uso de la tecnología y el aprendizaje organizacional sobre la satisfacción profesional de los residentes de pregrado.

\subsection{Satisfacción Profesional}

De acuerdo con Heslin (2005), satisfacción profesional es definida como una reflexión subjetiva que tiene una persona sobre su desarrollo profesional en un nivel individual. Judge, Cable, Boudreau y Bretz, (1995), considera que la satisfacción profesional está relacionada con el éxito profesional derivada a través de los resultados positivos que obtiene a través de experiencias de trabajo dentro de las organizaciones. 
Por otro lado, Abele y Spurk (2009), Abele et al. (2011) y Ng, Eby, Sorensen y Feldman (2005) consideran que la satisfacción profesional es un constructo en el que una persona evalúa a través de idiosincrasia individual su propia carrera, considerándola como un indicador central del éxito subjetivo en la carrera de la persona (Abele y Spurk, 2009; Greenhaus, Parasuraman y Wormley, 1990).

Por lo tanto, La satisfacción profesional es el placer que una persona se siente desde que un individuo logra determinadas metas (Kirkbesoglu y Ozder, 2015; Greenhaus et al., 1990). Asimismo, la satisfacción está ligado con otros indicadores internos de la organización, como el salario, la promoción que son ajenos por el individuo y que estos factores son evaluados por otras personas (Heslin, 2005). Sin embargo, existen otros factores externos que contribuyen al éxito o fracaso profesional, tal es el caso de las características personales del propio individuo que van configurando su vida empresarial y profesional que influyen en aspectos objetivos y en su vida privada (Kirkbesoglu y Ozder, 2015; Süral Özer y Tozkoparan, 2012).

Es menester considerar que existe una gran cantidad de variables que están dentro y fuera de las organizaciones que contribuyen en mejorar la satisfacción profesional. Incluso si el individuo busca tener un crecimiento vertical en la empresa donde labora, puede realzar un proceso que puede o no ser un progreso suficiente para el profesionista (Judge et al., 1995; Kirkbesoglu y Ozder, 2015).

Sin embargo, el grado de satisfacción profesional dependerá de su evaluación subjetiva a través que va satisfaciendo sus necesidades (Maslow, 1943) y logro de objetivos individuales que representes ser significativos en su profesión (Kirkbesoglu y Ozder, 2015). Es decir, la satisfacción profesional radica en que las personas han satisfecho sus necesidades y han alcanzado sus objetivos individuales a través de una evaluación subjetiva (Judge et al., 1995).

En este sentido, los residentes de pregrado considerados como parte de una generación llamada millenians, misma que, está fuertemente influenciada por el uso y manejo de la tecnología, resulta ser importante el emplear la tecnología como una herramienta que le permitirá satisfacer sus necesidades y lograr sus objetivos individuales y con ello, generará resultados positivos por medio del rendimiento organizativo tanto en lo individual como en lo grupal dentro de la organización. Finalmente, y a través de una evaluación subjetiva por parte del individuo, logrará una satisfacción profesional o de carrera 
en las organizaciones donde se encuentra realizando sus prácticas profesionales o laborando.

El constructo correspondiente a medir la satisfacción profesional es a través de la escala Career Satisfaction Scale (CSS), esta escala mide el grado de evaluación de una persona hacia el cumplimiento de diferentes metas como ingresos, crecimiento, desarrollo y objetivos general de la carrera, como también mide los éxitos globales relacionadas con su carrera (Abele y Spurk, 2009; Greenhaus et al., 1990).

A través del análisis teórico realizado a través de las variables estudiadas, se establecen las siguientes hipótesis:

\subsection{Hipótesis}

1. El uso de la tecnología influye positiva y significantemente sobre el aprendizaje organizacional de los residentes de pregrado

2. El uso de la tecnología influye positiva y significativamente sobre la satisfacción profesional de los residentes de pregrado.

3. El aprendizaje organizacional influye positiva y significativamente sobre la satisfacción profesional tecnología de los residentes de pregrado.

Para evaluar las hipótesis mencionadas anteriormente, se desarrolló un modelo de ecuación estructural hipotético (SEM). Ver figura 1

Figura 1. Modelo estructural hipotético de las variables exógenas y endógenas.

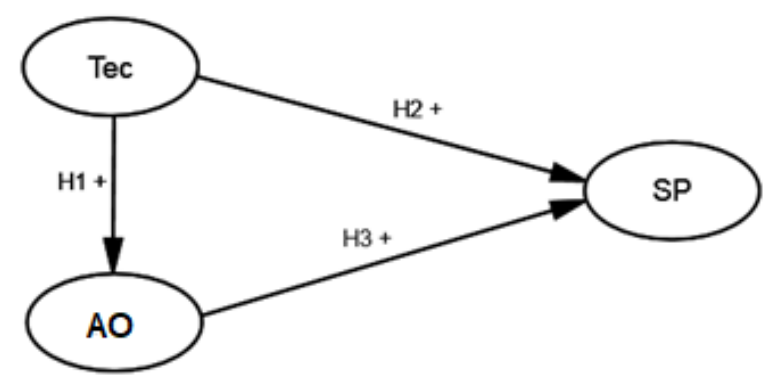

NOTA: Tec = Uso de la Tecnología, AO = Aprendizaje organizacional, SP = Satisfacción profesional . Fuente: Elaboración propia, 2017. 


\section{Metodología}

El enfoque de la presente investigación fue cuantitativo y explicativo, debido a las características de medición que presentan las variables elegidas, mismas que pretenden, dar una visión general y aproximada a una determinada realidad, en este sentido, la satisfacción profesional de los residentes de pregrado. Así como también fue observacional, ya que se pretendió describir el fenómeno, sin realizar ninguna intervención o manipular las variables que determinaron el proceso de la investigación. El tipo de estudio fue transversal, en razón al periodo y secuencia del estudio, para ello se aplicaron instrumentos en una sola ocasión, en un mismo momento a los sujetos del estudio, es decir, se realizó un corte en el tiempo, con la finalidad de obtener la información necesaria para el análisis y medición de las variables. Así mismo, el nivel de significancia establecido para aceptar las hipótesis establecidas fue al $95 \%(p<=0.05)$

\subsection{Muestra}

El tipo de muestreo fue no probabilístico intencional, debido a que se requería obtener el mayor número de participantes, en cuanto a los criterios de inclusión y exclusión de la muestra fueron hombres y mujeres residentes de universidades públicas. La muestra fue conformada por 220 residentes de universidades públicas del estado de Guanajuato, de los cuales, el $38.6 \%$ eran varones mientras que el $61.4 \%$ fueron mujeres. Con respecto a la edad de los sujetos el $64.1 \%$ cuenta con una edad menor a 22 años, mientras que el $30.9 \%$ tiene una edad de 23 a 25 años., $3.2 \%$ cuenta con una edad de 26 a 28 años, el .5\% tiene una edad de 29 a 30 años y finalmente, el $1.4 \%$ tiene una edad de más de 30 años. Con respecto a la carrera profesional, el 1.4\% son residentes de la Ingeniería en Bioquímica, el 7.3\% son residentes de la Ingeniería de Electromecánica, el $5 \%$ son residentes de la Ingeniería de Industrial, el 8.2\% son residentes de la Ingeniería de Informática, 53.6\% son residentes de la Ingeniería en Gestión empresarial, el 20.9\% son residentes de la Ingeniería de Logística y transporte y finalmente, el 3.6\% la Licenciatura en Administración

Para el análisis de los datos obtenidos, se empleó el software estadístico SPSS Statistics v.20 y para la comprobación de las hipótesis establecidas, se desarrolló un modelo de ecuación estructural (SEM), a través del software estadístico Mplus v.7.0.

Asimismo, se procedió a realizar una correlación entre las variables latentes estudiadas. De esta manera, se demuestra que existe una relación positiva y significativa 
(Bonett y Wright, 2000; Pearson, 1929, 1931) entre las variables latentes: uso de la tecnología, el rendimiento organizativo y la satisfacción profesional de los residentes de pregrado, medida a través del coeficiente de correlación de Pearson como se muestra en la tabla 1.

Tabla. 1. Estadísticos descriptivos de las variables latentes.

\begin{tabular}{|c|c|c|c|c|c|c|}
\hline VARIABLES & Media & D.S. & Varianza & Tec & AO & SP \\
\hline Uso de la Tecnología & 5.20 & 0.901 & 0.812 & 1.000 & & \\
\hline Aprendizaje Organizacional & 5.79 & 1.12 & 1.27 & $.416^{\star \star}$ & 1.000 & \\
\hline Satisfacción Personal & 7.31 & 1.38 & 1.38 & $.400^{\star *}$ & $.612^{\star *}$ & 1.000 \\
\hline
\end{tabular}

\subsection{Instrumentos}

\subsubsection{Uso de la Tecnología}

Para medir este constructo de los residentes de las universidades, se empleó el instrumento Uso de las Tic's en las Organizaciones desarrollado por Siddiq, F., Gochyyev, P. y Wilson, M. (2017). Este instrumento está conformado por 6 ítems. Los reactivos tienen un formato tipo Likert con 6 puntos de repuesta, donde 1 representa "Totalmente en desacuerdo" y 6 "Totalmente de acuerdo". Para evaluar la confiabilidad de este instrumento se empleó el Alfa de Cronbach ( $\alpha=.85)$ así como la confiabilidad compuesta $(\rho c=.86)$ de Dillon-Goldstein, mismas que resultaron satisfactorias (Cronbach, 1951; Hair, Hult, Ringle y Sarstedt, 2014). Para medir el constructo del Uso de la Tecnología se obtuvo a través de una puntuación media de todos los ítems que conforma la escala

En cuanto a la validez del Uso de la Tic's en las Organizaciones, se desarrolló un análisis factorial confirmatorio (CFA) basado en Siddiq, F., Gochyyev, P. y Wilson, M. (2017) a través de un modelo de medida de constructo . Para la validación del SEM, se consideró la prueba de Chi-cuadrado ( $\left.\chi^{2}=12.48 / g /=5\right)$, el índice comparativo de ajuste ( $\left.\mathrm{CFl}=.987\right)$, el índice de Tucker-Lewis (TLI=.960), Índice de la Aproximación de la Raíz Cuadrada del Error Cuadrático Medio (RMSEA=.08), por lo que los índices de bondad y ajuste del modelo resultaron ser satisfactorios como se muestra en la tabla 2. 
Tabla 2. Cargas factoriales estandarizadas de los ítems con relación al factor Uso de la Tecnología e índices del análisis factorial confirmatorio.

USO DE LA TECNOLOGÍA

\begin{tabular}{|c|c|c|c|c|}
\hline \multicolumn{3}{|c|}{ VARIABLE } & CARGA & RESIDUAL \\
\hline \multicolumn{3}{|c|}{$\begin{array}{l}\text { 1.-Puedo trabajar mediante el uso de la tecnología en mi centro de } \\
\text { trabajo }\end{array}$} & 0.675 & 0.545 \\
\hline \multicolumn{3}{|c|}{ 2.-Desarrollo mejor mi trabajo si utilizo herramientas tecnológicas. } & 0.829 & 0.312 \\
\hline \multicolumn{3}{|c|}{$\begin{array}{l}\text { 3.-Tengo la capacidad de utilizar la tecnología que requieren las } \\
\text { empresas }\end{array}$} & 0.610 & 0.628 \\
\hline \multicolumn{3}{|c|}{$\begin{array}{l}\text { 4.-Necesito tener habilidades sobre el manejo de la computadora } \\
\text { para conseguir el trabajo que quiero }\end{array}$} & 0.593 & 0.648 \\
\hline \multicolumn{3}{|c|}{$\begin{array}{l}\text { 5.-El uso de la tecnología es importante para realizar mi trabajo más } \\
\text { eficiente }\end{array}$} & 0.795 & 0.367 \\
\hline \multicolumn{3}{|c|}{$\begin{array}{l}\text { 6.-Necesito tener habilidades tecnológicas para ingresar o continuar } \\
\text { laborando en mi centro de trabajo. }\end{array}$} & 0.745 & 0.445 \\
\hline CFI & RMSEA & CONF. CC & & $\alpha$ - CRONBACH \\
\hline 0.987 & 0.08 & 0.02 & & 0.85 \\
\hline
\end{tabular}

Fuente: Siddiq, F., Gochyyev, P. y Wilson, M. (2017).

\subsubsection{Aprendizaje Organizacional}

Para medir este constructo se empleó la escala de Bontis et al. (2002). Este instrumento está conformado un total de 6 ítems. Así mismo, emplea una escala de Likert de 7 puntos, donde 1 representa "En Total desacuerdo" y 7 "En Total acuerdo". Para evaluar la confiabilidad de la escala ( $\alpha=.91$ ), empleó el Alfa de Cronbach, así como la confiabilidad compuesta $(\rho c=.94)$ de Dillon-Goldstein misma que resultó satisfactorio (Cronbach, 1951; Hair et al., 2014). Para medir el constructo del Aprendizaje Organizacional se obtuvo a través de una puntuación media de todos los ítems que conforma la escala

En cuanto a la validez de la escala de negociación, se desarrolló un CFA basado en Bontis et al. (2002), a través de un modelo de ecuación estructural. Para la validación del modelo de medición del constructo, se consideraron los índices de bondad y ajuste del modelo $(\mathrm{CFI}=1.000 ; \mathrm{TLI}=.999$; $\mathrm{RMSEA}=0.01$; $\mathrm{SRMR}=0.007)$, por lo que resultaron ser satisfactorios (Bollen, 1989; Jöreskog y Sörbom, 1981; Muthén y Muthén, 1998-2007; Muthén, 2001, 2002; Rigdon, 1996). Los resultados se muestran en la tabla 3. 
Tabla 3. Cargas factoriales estandarizadas de los ítems con relación al factor Aprendizaje Organizacional e índices del análisis factorial confirmatorio.

APRENDIZAJE A NIVEL ORGANIZACIONAL

\begin{tabular}{|c|c|c|c|c|c|}
\hline \multicolumn{4}{|c|}{ VARIABLE } & CARGA & RESIDUAL \\
\hline \multirow{2}{*}{\multicolumn{4}{|c|}{$\begin{array}{l}\text { 1.-Contamos con una estrategia que nos posiciona bien en el } \\
\text { futuro } \\
\text { 2.-La estructura organizacional apoya nuestra dirección } \\
\text { estratégica }\end{array}$}} & 0.840 & 0.294 \\
\hline & & & & 0.861 & 0.259 \\
\hline \multicolumn{4}{|c|}{$\begin{array}{l}\text { 3.-La estructura organizacional nos permite trabajar de 4.- } \\
\text { manera efectiva }\end{array}$} & 0.826 & 0.318 \\
\hline \multicolumn{4}{|c|}{$\begin{array}{l}\text { 4.-Nuestros procedimientos operativos nos permiten trabajar } \\
\text { de manera eficiente }\end{array}$} & 0.868 & 0.247 \\
\hline CFI & TLI & RMSEA & SRMR & CONF. COMP. & $\alpha-$ CRONBACH \\
\hline 1.000 & 0.999 & 0.01 & 0.007 & 0.945 & 0.91 \\
\hline
\end{tabular}

Fuente: Bontis et al. (2002)

\subsubsection{Satisfacción Profesional}

Para medir este constructo se empleó la escala de satisfacción profesional (CSS: Career Satisfaction Scale) desarrollada por Greenhaus, Parasuraman y Wormley (1990). De esta escala se consideraron dos dimensiones, el rendimiento a nivel individual y el rendimiento a nivel grupal. Este instrumento está conformado un total de 5 ítems. Así mismo, emplea una escala de Likert de 9 puntos, donde 1 representa "Fuertemente en desacuerdo" y 7 "Totalmente de acuerdo". Para evaluar la confiabilidad de la escala $(\alpha=.92)$, empleó el Alfa de Cronbach, así como la confiabilidad compuesta $(\rho c=.92)$ de Dillon-Goldstein misma que resultó satisfactorio (Cronbach, 1951; Hair et al., 2014). Para medir el constructo del Satisfacción Profesional se obtuvo a través de una puntuación media de todos los ítems que conforma la escala

En cuanto a la validez de la escala, se desarrolló un CFA basado en Greenhaus, Parasuraman y Wormley (1990), a través de un modelo de medición del constructo. Para la validación del SEM, se consideraron los índices de bondad y ajuste del modelo $(\mathrm{CFI}=.999$; $\mathrm{TLI}=.987$; RMSEA=0.07; SRMR=0.007), por lo que resultaron ser satisfactorios (Bollen, 1989; Jöreskog y Sörbom, 1981; Muthén y Muthén, 1998-2007; Muthén, 2001, 2002; Rigdon, 1996). Esto se muestra en la tabla 4. 
Tabla 4. Cargas factoriales estandarizadas de los ítems con relación al factor Satisfacción Profesional e índices del análisis factorial confirmatorio

SATISFACCIÓN PROFESIONAL

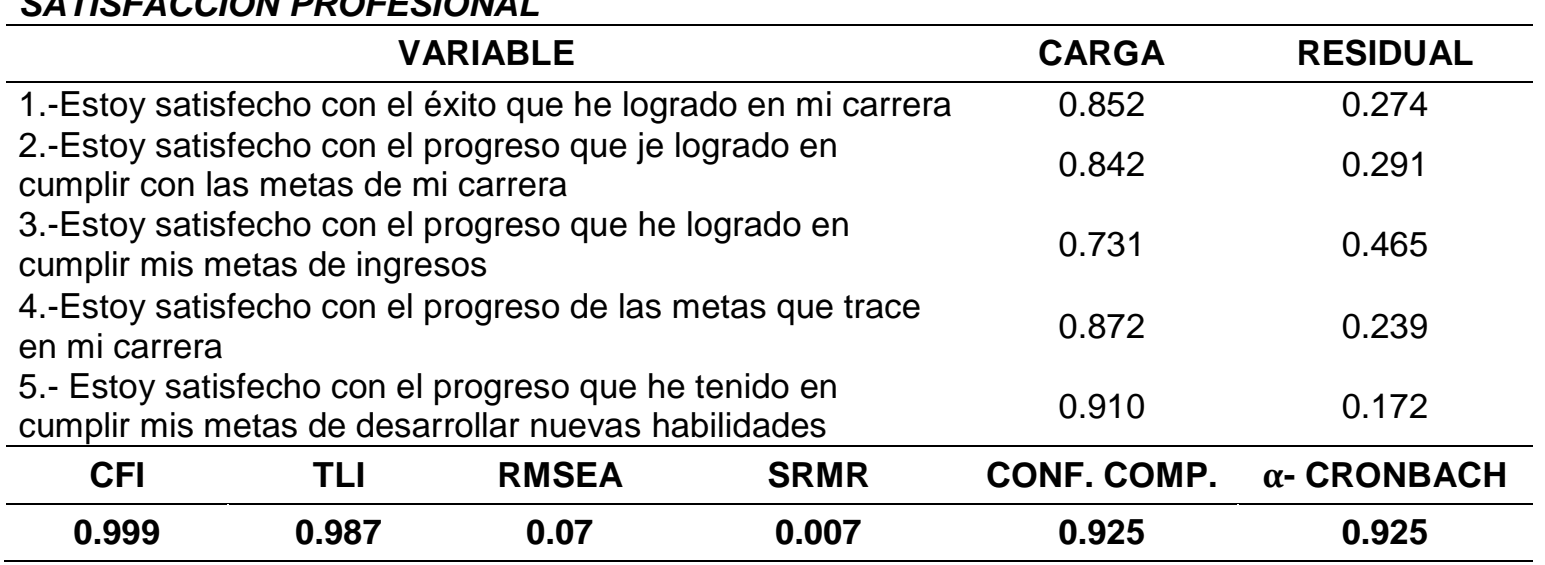

Fuente: Greenhaus, Parasuraman y Wormley (1990).

\section{Resultados}

Para evaluar el modelo de medición de constructo hipotético puesto en cuestión (SEM), se consideraron los siguientes índices de bondad y ajuste: Chi-cuadrado $\left(\chi^{2}=137.575 \mathrm{gl}=\right.$ 84), por lo que la prueba de Chi-cuadrada $\left(\chi^{2} / g l=1.63 ; p<0.05\right)$ resultó ser satisfactoria, el Índice de Ajuste Comparativo (CFI = .976 y TLI=.970) así como la Aproximación de la Raíz Cuadrada del Error Cuadrático Medio $(R M S E A=.05)$ y finalmente, la Raíz Cuadrada Media Residual Estandarizado (SRMR=0.03), por lo el modelo resultó ser absolutamente deseable y aceptable (Jöreskog y Sörbom, 1981; Muthén y Muthén, 1998-2007; Rigdon, 1996). Ver Figura 2.

Figura 2. Cargas estructurales estandarizadas del modelo hipotético puesto en cuestión.

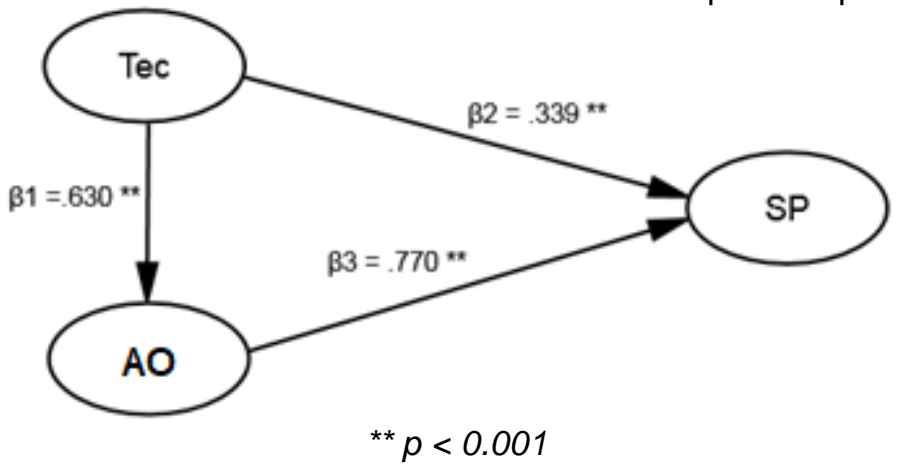

NOTA: $\mathrm{Tec}=$ Uso de tecnología, $\mathrm{AO}=$ =Aprendizaje Organizacional, SP =Satisfacción Profesional. Fuente: Elaboración propia, 2017. 
Los resultados obtenidos mediante el modelo hipotético de ecuación estructural (ver Figura 2), demuestran que existe evidencia estadística para afirmar que, el uso de la tecnología (Tec) tiene un efecto positivo y significativo $(\beta 1=.630 ; p<0.001)$ sobre el aprendizaje organizacional $(\mathrm{AO})$ de los residentes de pregrado en las empresas del sector industrial. Por lo que, se acepta la hipótesis H1. En este sentido, se cuenta con suficiente evidencia estadística para afirmar que el uso y manejo de la tecnología influye sobre el rendimiento organizativo en los residentes de pregrado en las empresas del sector industrial.

En este sentido, los residentes cuentan con altas habilidades y destrezas en el uso del manejo de tecnología lo que contribuye de manera positiva en las organizaciones a generar un proceso de desarrollar nuevas tendencias para la toma de decisiones, innovando los procesos de gestión, generar acciones y medidas eficientes y eficaces por medio de grupos de colaboración de manera que se genere un aprendizaje continuo en las organizaciones.

Así mismo, se demuestra con bases estadísticas que, el uso de la tecnología (Tec) tiene un efecto positivo y significativo $(\beta 2=.339 ; \mathrm{p}<0.001)$ sobre la satisfacción profesional (SP) de los residentes de pregrado en las empresas del sector industrial. Por lo que la hipótesis $\mathrm{H} 2$ es aceptada. En este sentido, se cuenta con suficiente evidencia estadística para afirmar que el uso y manejo de la tecnología de los residentes de pregrado influye positiva y significativamente en su satisfacción profesional.

Por lo tanto, el residente emplea de manera eficaz el uso de la tecnología para el desarrollo de las actividades dentro del en el sector productivo y empresarial, por lo que genera en él una satisfacción profesional a la carrera que ha estudiado, debido a que, relaciona lo aprendido profesionalmente con el contexto en ámbito laboral en el que se desenvuelve conduciendo lo al éxito profesional a través del logro de metas tanto personales y colectivas a través de la organización de la cual colabora, generándole nuevas áreas de oportunidad de las cuales puede ser explotadas por el mismo residente, en el que puede contribuir a ser más competitiva la empresa

Finalmente, para evaluar la hipótesis H3 se consideró el modelo estructural hipotético (ver Figura 2), en la que se cuenta con suficiente evidencia estadística para afirmar que el aprendizaje organizacional $(A O)$ influye positiva y significativamente $(\beta 3=.770 ; p<0.001)$ sobre la satisfacción profesional (SP) en los residentes de pregrado en las empresas del sector industrial. En este sentido, se acepta la hipótesis H3. 
Es por ello que, es importante generar y establecer un proceso de generación de nuevas ideas, conocimientos, procedimientos, reglas y estrategias así como comportamientos con la finalidad de poder resolver los diferentes tipos de eventualidades que enfrenta las empresas, es por ello que los residentes juegan un papel importante pues es ellos cuentan con las habilidades del uso de la tecnología en beneficio de obtener buenos resultados a la organización de manera que les generen una satisfacción profesional, debido que esta satisfacción es una motivación tanto extrínseca como intrínseca que promoverá e los residentes a convertir a las organizaciones más competitivas y vanguardistas factores necesarios para enfrentar un mundo globalizado por las que toda empresa enfrenta día a día logrando a través de los residentes el éxito.

Es importante señalar que ese éxito organizacional es permeable pues, se generara satisfacción y éxito en lo colectivo e individual de los residentes y con ello el éxito de la carrera profesional del que el residente opto por estudiar y, en ese sentido, el éxito de la universidad, debido que la visión de estas está enfocada en ser reconocidas a nivel estatal o nacional como una de las instituciones educativas de mayor prestigio y eso es logrado no solamente a través del posicionamiento de sus egresados en el sector productivo sino también a través de la satisfacción profesional que los residentes tienen al momento de introducirse en el mundo laboral.

\section{Conclusiones}

Ante los resultados obtenidos a través de la presente investigación, podemos darnos cuenta de que las organizaciones atraviesan por diversos escenarios generados por la globalización por un lado y por otro la influencia que ejerce las nuevas generaciones, ambos marcados por el uso y manejo de la tecnología.

Es por ello que, los residentes de pregrado de las diferentes universidades aplican sus conocimientos, habilidades que les fueron adquiridos a través de su formación académica y en muchas de las ocasiones empata con el uso tecnológico de las organizaciones logrando los objetivos personales y organizacionales, en este sentido, se van desarrollando profesionalmente alcanzando el éxito y por ende una satisfacción profesional.

Por otro lado, surgen nuevas áreas de oportunidad en el ámbito empresarial, a razón de que actualmente se cuenta con recurso humano que está fuertemente influenciada por la tecnología, es decir, que no tiene dificultades en usarla, manejarla para automatizar 
procesos tanto administrativos, producción o de gestión logrando en ellos sus objetivos a nivel individual y grupal. En este sentido, el que una organización cuente con personal con habilidades en el uso y manejo de tecnología influirá en el aprendizaje organizacional, en este sentido, representa una ventaja competitiva (Hong, 1999; O'Keeffe, 2003) de la misma empresa hacia sus competidores.

En este sentido, el uso de la tecnología juega un papel muy importante dentro del sector empresarial, y ante esta nueva generación, representa una nueva estrategia de las organizaciones que pueden aprovechar estos factores que se presentan, pues al conjuntarlos, representa una ventaja competitiva en el mercado en que se desenvuelve la empresa.

Los alcances obtenidos con la presente investigación consistieron en la identificación de la influencia conjunta del uso de la tecnología y el aprendizaje organizacional en relación con la satisfacción profesional en los residentes de pregrado de universidades públicas. Las limitaciones referidas en la investigación fueron establecidas a través de la metodología, el procedimiento en la recolección de datos y el análisis de los datos y finalmente las limitaciones teóricas. Es decir, el enfoque de la investigación se llevó a cabo bajo un paradigma cuantitativo, explicativo, observacional y transversal.

Cabe señalar el uso de la tecnología juega un papel importante en el aprendizaje organizacional, pues la organización se ve beneficiada a través de nuevos enfoques y procesos que la organización adapta por medio de las residencias o prácticas profesionales de los alumnos universitarios de pregrado que se van penetrando en el sector productivo. Así mismo, le genera una satisfacción de lo que ellos estudiaron permeándoles experiencia, así como una diversidad de habilidades y atributos para el desarrollo de su profesión en el ámbito laboral.

\section{Referencias}

Abele, Andrea y Spurk, Daniel. (2009). The longitudinal impact of self-efficacy and career goals on objective and subjective career success. Journal of Vocational Behavior, 74(1), 53-62. Doi: https://doi.org/10.1016/i.jvb.2008.10.005

Antonacopoulou, Elena. (2006). The Relationship between Individual and Organizational Learning: New Evidence from Managerial Learning Practices. Management Learning. $37(4)$, 455-473. Recuperado http://journals.sagepub.com/doi/abs/10.1177/1350507606070220 
Assar, Saïd. (2015). Information and Communications Technology in Education. En James D. Wright (Ed.), International Encyclopedia of the Social y Behavioral Sciences, (2 ${ }^{\text {nd }}$ ed.; pp. 66-71). Recuperado de https://www.researchgate.net/publication/277669538 Information and Communication $\underline{\mathrm{s} \text { Technology in Education }}$

Barakat, Areeg y Moussa, Faten. (2014). Variables influencing expatriate learning and organizational learning. Competitiveness Review, 24(4), 275-292. doi: https://doi.org/10.1108/CR-06-2013-0063

Bollen, Kenneth A. (1989). Structural equations with latent variables. New York, NYS: John Wiley y Sons.

Bonett, Douglas y Wright, Thomas. (2000). Simple size requirements for estimating Pearson, Kendall and Sperman correlations. Psichometrika, 65(1), 23-28. doi: https://doi.org/10.1007/BF02294183

Bontis, Nick, Hulland, John y Crossan, Mary. (2002). Managing an organizational learning system by aligning stocks and flows. Journal of Management Studies, 39, 437-469. doi:10.1111/1467-6486.t01-1-00299.

Clark, Sue Campbell. (2003). Enhancing the educational value of business internships. Journal of Management Education, 27(4), 472-484. Recuperado de http://journals.sagepub.com/doi/abs/10.1177/1052562903251350

Coco, M. (2000). Internships: A try before you buy arrangement. SAM Advanced Management Journal, 65, 41-43.

Compaine, Benjamin. (2001). The Digital Divide: Facing a Crisis or Creating a Myth? Cambridge, MA: Mit-press.

Cronbach, Lee. (1951). Coefficient alpha and internal structure of test. Psychometrica, 16, 297-335. doi: http://dx.doi.org/10.1007/BF02310555

De La Garza, María, Zavala, María y López-Lemus, Jorge. (2017). Competencias del emprendedor y su impacto en el desempeño organizacional. Universidad y Empresa. 19 (33), 53-74. Doi: 10.12804/revistas.urosario.edu.co/empresa/a.4811

El-Temtamy, Osama, O'Neill, Kathleen, y Midraj, Sadiq. (2016). Undergraduate employability training and employment: a UAE study. Higher Education, Skills and Work-Based Learning, 6(1), 100-115. doi: 10.1108/HESWBL-02-2015-0006

Gamino, Carranza, Arturo, Acosta González, Mara Grassiel, y Pulido Ojeda, Rocío Elizabeth. (2016). Modelo de formación dual del Tecnológico Nacional de México. Revista de Investigación en Educación, 14(2), 170-183. Recuperado de https://dialnet.unirioja.es/servlet/articulo?codigo=5698034 
García Lirios, Cruz. (2013). La red de conocimiento en una universidad con sistema de prácticas profesionales y servicio social tecnológico-administrativo. Fundamentos en Humanidades, 14(27), 135-157.

Gómez, Denisse (Coord.). (2011). Prospectiva e Innovación Tecnológica. México: Editores Siglo XXI.

Greenhaus, Jeffrey, Parasuraman, Saroj y Wormley, Wayne. (1990). Effects of Race on Organizational Experiences, Job Performance Evaluations, and Career Outcomes. The Academy of Management Journal, 33(1), 64-86.

Gurau, Călin. (2012). A life-stage analysis of consumer loyalty profile: comparing Generation $X$ and Millennial consumers. Journal Consumer Mark, 29(2), 103-13. Doi: $10.1108 / 07363761211206357$

Gursoy, Dogan, Geng-Qing, Christina y Karadag, Ersem. (2013). Generational differences in work values and attitudes among frontline and service contact employees. International Journal of Hospitality Management, 32(1), 40-48. doi: 10.1016/j.jjhm.2012.04.002.

Hair, Joseph Hult, Thomas, Ringle, Christian y Sarstedt, Marko. (2014). A primer on partial least squares structural equation modeling (PLS SEM). USA: SAGE Piblications.

Hershatter, Andrea y Epstein, Molly. (2010). Millennials and the world of work: An organization and management perspective. Journal of Business and Psychology, 25(2), 211-223. Doi: 10.1007/s10869-010-9160-y

Heslin, Peter. (2005). Conceptualizing and evaluating career success. Journal of Organizational Behaviour, 26 (2), 113-136. doi: http://dx.doi.org/10.1002/job.270

Hirsch, Julia, Rodríguez, Ignacio y Manríquez, Martha. (2015). La preparación de las empresas manufactureras del Estado de Querétaro, México, en el área de las tecnologías de información y comunicación. Suma de Negocios, 6(14), 1-12. doi. 10.1016/j.sumneg.2015.08.012.

Hofstede, Geert. (1980). Culture’s Consequences. Beverly Hills, CA: Sage.

Hong, Jacky. (1999). Structuring for organizational learning. The Learning Organization, 6(4), 173-186. doi:10.1108/09696479910280631

Jöreskog, Karl, y Sörbom, Dag. (1981). LISREL: Analysis of linear structural relationships by maximum likelihood and least squares methods. Chicago, IL: National Educational Resources.

Judge, Timothy, Cable, Daniel, Boudreau, John y Bretz, Robert. (1995). An empirical investigation of the predictors of executive career success. Personnel Psychology, 48(3), 485-519. Recuperado: http://dx.doi.org/10.1111/j.1744-6570.1995.tb01767.x

Kim, Daniel. (1993). The link between individual and organizational learning. Recuperado de http://www.iwp.jku.at/born/mpwfst/03/0312 ivkim.pdf 
Kirkbesoglu, Erdem y Ozder, Emir. (2015). The Effects of Organizational Performance on the Relationship between Perceived Organizational Support and Career Satisfaction: An Application on Insurance Industry. Journal of Management Research, 7(3), 35-50. doi: 10.5296/jmr.v7i3.7094

Knouse, Stephen, B., y Fontenot, Gwen. (2008). Benefits of the business college internship: A research review. Journal of Employment Counseling, 45(2), 61-66. doi: 10.1002/j.2161-1920.2008.tb00045.x

Kupperschmidt, Betty. (2000). Multigeneration employees: strategies for effective management. The Health Care Manager, 19(1), 65-76. Doi: 10.1097/00126450200019010-00011

LeBrasseur, Rolland, Whissell, Robert y Ojha, Abhoy. (2002). Organizational learning, transformational leadership and implementation of continuous quality improvement in Canadian hospitals. Australian Journal of Management, 27(2), 141-162. doi: 10.1177/031289620202700203

Lee, Tae y Kim, Jeong. (2014). Residents respomses on indoor environment quality and energy use in apartments. Energy and Buildings, 98(1), 34-38. Doi: 10.1016/j.enbuild.2014.10.084.

Lissitsa, Sabina y Kol, Ofrit. (2016). Generation X vs. Generation Y - A decade of online shopping. Journal of Retailing and Consumer Services, 31, 304-312. Doi: 10.1016/j.jretconser.2016.04.015.

López-Lemus, Jorge, De La Garza, María y Zavala, María. (2016). The Leadership Influence of Entrepreneurs in the Results of SMEs. International Journal of Business and Social Science, $7(11), 40-50$.

Lowden, K., Hall, S., Elliot, D. y Lewin, J. (2011). Employers' perceptions of the employability skills of new graduates. University of Glasgow SCRE Centre and Edge Foundation. London, Uk.

Maslow, Abraham. (1943). A theory of human motivation. Psychological review, 50(4), 370.

Miller, Linda, Biggart, Andy y Newton, Becci. (2013). Basic and employability skills. International Journal of Training and Development, 17(3), 173-175. doi: 10.1111/ijtd.12007

Muthén Bengt. (2001). Second-Generation structural equation modeling with a combination of categorical latent variables: New opportunities for latent class/latent growth modeling. En Linda M. Collins y Aline Sayer (Eds.), New Methods for the Analysis of Change (pp. 289-332). Washington, D.C.

Muthén, Bengt. (2002). Beyond SEM: General latent variable modeling. Behaviometrika, 29(1), 81-117. Doi: 10.2333/bhmk.29.81 
Muthén, Linda y Muthén Bengt. (1998-2007). Mplus Version 5.0 statistical analysis with latent variables: User's Guide (4 ${ }^{\text {th }}$ ed.). Los Angeles, CA: Muthen y Muthen.

$\mathrm{Ng}$, Thomas, Eby, Lilian, Sorensen, Kelly y Feldman, Daniel. (2005). Predictors of objective and subjective career success. A meta-analysis. Personnel Psychology, 58, 367-408. doi: 10.1111/j.1744-6570.2005.00515.x

Norum, Pamela. (2003). Examination of generational differences in household apparel expenditures. Family y Consumer Sciences Research Journal, 32(1), 52-75, doi: 10.1177/1077727X03255901.

O'Keeffe, Ted. (2003), Preparing expatriate managers of multinational organizations for the cultural and learning imperatives of their job in dynamic knowledge-based environments. Journal of European Industrial Training, 27(5), 233-243. Doi: $10.1108 / 03090590310469614$

Süral Özer, Pinar y Tozkoparan, Güler. (2012). An Empirical Study of the Relationship among Core Self Evaluations with Job and Life Satisfaction. Journal of Yasar University, 30(8), 4933-4947.

Recuperado

de

http://eds.a.ebscohost.com/eds/detail/detail?vid=0\&sid=ec0f88d1-4777-4c28-9befd8d6d99ccd6d\%40sessionmgr4007\&bdata=Jmxhbmc9ZXMmc2I0ZT1|ZHMtbGI2ZSZzY 29wZT1zaXR|\#AN=90599702\&db=a9h

Parment, Anders. (2013). Generation Y vs. Baby Boomers: shopping behavior, buyer involvement and implications for retailing. Journal Retailing and Consumer Services, 20(2), 189-199. Doi: 10.1016/j.jretconser.2012.12.001.

Pearson, E. (1929). Some notes on sampling tests with two variables. Biometrika, 21(1/4), 337-360. Doi: $10.2307 / 2332565$

Pearson, E. (1931). The test of significance for the correlation coefficient. Journal of the American Statistical Association, 26, 128-134. doi: 10.1080/01621459.1931.10503208

Porter, Michael. (2001). Strategy and the Internet. Harvard Business Review, 79(3), 63-78.

Prior, Jason, Hubbard, Phil y Rai, Tapan. (2015). Using residents' worries about technology as a way of resolving environmental remediation dilemmas. Science of total environment, 580, 882-899. doi: https://doi.org/10.1016/j.scitotenv.2016.12.035

Rees, Claire, Forbes, Peter y Kubler, Bianca. (2006). Student employability profiles: a guide for higher education practitioners. Recuperado de: https://www.heacademy.ac.uk/system/files/student employability profiles apr07.pdf

Rigdon, Edward. (1996). CFI versus RMSEA: A comparison of two fit indexes for structural equation modeling, Structural Equation Modeling: A Multidisciplinary Journal, 3(4), 369379. doi: 10.1080/10705519609540052 
Sánchez de Mantrana, Mirna. (2005). El aprendizaje en contextos laborales reales: el caso de las pasantías de los estudiantes universitarios. Educere, 9(30), 345-357. Recuperado de: http://www.redalyc.org/articulo.oa?id=35603010

Secretaria de Educación Pública (SEP) y Subsecretaría de Educación e Investigación Tecnológica (SEIT). (1997). Manual de procedimientos para la planeación, operación y acreditación de las residencias profesionales en los institutos tecnológicos. Recuperado de http://www.itver.edu.mx/index.php/normateca/documentosoperativos/manuales

Secretaria de Educación Pública, México (SEP). (2017). Tecnológico Nacional de México: Residencias profesionales. Recuperado de http://itcelaya.edu.mx/index.php?r=residencias

Senge, P. (1990). The fifth Discipline: The Art and Practice of the Learning Organisation. New York: Doubleday Currency.

Siddiq, Fazilat, Gochyyev, Perman y Wilson, Mark. (2017). Learning in Digital Networks - ICT Literacy: A Novel Assessment of Students' 21st Skills. Computers y Education, 109, 11-37. doi: 10.1016/j.compedu.2017.01.014.

Strauss, William y Howe Neil. (1991). Generations: The History of America's Future. Quill William Morrow, New York, pp. 1584-2089.

Wong, May M.L. (2001), Internationalizing Japanese expatriate managers; organizational learning through international assignment. Management Learning, 32(2), 237-251. doi: $10.1177 / 1350507601322005$ 